\title{
A Study on the Prediction of Reproductive Outcomes in Frozen Embryo Transfer Cycles by Calculating the Volume of Uterine Junctional Zone with Three-Dimensional Ultrasound
}

\author{
Vorhersage des reproduktiven Outcomes bei Transfer im Kryozyklus \\ (Frozen Embryo Transfer) durch Berechnung des Volumens des \\ Endometriums der Junktionalzone mit 3D-Ultraschall
}

(c) (ㅇ) $९$

Authors

Yan Liu', ${ }^{1}$, Lei Wang ${ }^{3}$, MeiXian Wang ${ }^{3}$, Yu Jiang1', TingTing Xia ${ }^{3}$, QingXiong Yue ${ }^{4}$, Yujia Sha ${ }^{4}$, Ying Huang ${ }^{2}$

Affiliations

1 Department of Ultrasound, Dalian Municipal Women and Children's Medical Center, Dalian, China

2 Department of Ultrasound, Shengjing Hospital of China Medical University (85024), Shenyang, China

3 Department of Reproductive Center, Dalian Municipal Women and Children's Medical Center, Dalian, China

4 Department of Ultrasound, Dalian Municipal Central Hospital Affiliated of Dalian Medical University (74545), Dalian, China

Key words

three-dimensional ultrasound, endometrial volume, in vitro fertilization, clinical pregnancy, uterine junctional zone

received 29.05.2021

accepted 31.08.2021

published online 24.11.2021

Bibliography

Ultraschall in Med 2023; 44: e126-e135

DOI 10.1055/a-1634-4955

ISSN $0172-4614$

(C) 2021. The Author(s).

This is an open access article published by Thieme under the terms of the Creative Commons Attribution-NonDerivative-NonCommercial-License, permitting copying and reproduction so long as the original work is given appropriate credit. Contents may not be used for commercial purposes, or adapted, remixed, transformed or built upon. (https://creativecommons.org/licenses/by-nc-nd/4.0/).

Georg Thieme Verlag KG, Rüdigerstraße 14,

70469 Stuttgart, Germany

Correspondence

Ying Huang

Shengjing Hospital of China Medical University

Department of Ultrasound, No. 36, Sanhao Street, Heping

District, 110004 Shenyang, China

huangying712@163.com

\section{ABSTRACT}

Purpose To prospectively study the influence of the volume of the uterine junctional zone (JZ) as a novel predictor of reproductive outcomes in frozen embryo transfer cycles.

Methods Among the first 30 patients, intra- and interobserver repeatability was evaluated and expressed as a coefficient of repeatability. The same classification system was used to evaluate the JZ of 142 infertility patients undergoing in vitro fertilization (IVF). Ultrasonography was performed on the day before transplantation. The three-dimensional (3D) volume images were then analyzed to obtain the volume of the endometrium (EV), the average thickness of the JZ on the coronal plane, and the volume of the JZ (JZV). The JZV was then divided by the EV. These parameters were compared with the outcomes of clinical pregnancy.

Results The $3 \mathrm{D}$ image showed that the $\mathrm{JZ}$ achieved a good intra- and interobserver consistency $(k=0.862, k=0.694)$. The total pregnancy rate was $47 \%$. There was a highly significant difference between pregnant and non-pregnant women with respect to age $(p<0.001), J Z V(p=0.003)$, and JZV/EV $(p<0.001)$ on the day before transplantation. Age and JZV/ $E V$ were independent factors for predicting the success of IVF transplantation $(p=0.010, p=0.016)$. The area under the ROC curve of JZV/EV in predicting clinical pregnancy was 0.688 , the cut-off value was 0.54 , the sensitivity was $83.8 \%$, and the specificity was $50.0 \%$.

Conclusion Age and JZV/EV are independent factors for predicting the success of frozen embryo transfer cycles in IVF. A smaller JZV/EV was more beneficial for clinical pregnancy.

\section{ZUSAMMENFASSUNG}

Ziel Prospektive Untersuchung des Einflusses des Volumens der Junktionalzone (JZ) des Myometriums als neuartiger Prädiktor für den reproduktiven Outcome nach Transfer im Kryozyklus (Frozen-Embryo-Transfer, FET).

Methoden Bei den ersten 30 Patientinnen wurde die Intraund Interobserver-Reproduzierbarkeit bewertet und die Wiederholbarkeit als „Coefficient of Repeatability“ ausgedrückt. 
Dasselbe Klassifizierungssystem wurde zur Bewertung der JZ von 142 Patientinnen mit Infertilität verwendet, die sich einer In-vitro-Fertilisation (IVF) unterzogen. Die Ultraschalluntersuchung wurde am Tag vor dem Transfer durchgeführt. Die 3dimensionalen (3 D) Volumenbilder wurden anschließend analysiert, um das Endometriumvolumen (EV), die durchschnittliche Dicke der JZ in der koronalen Ebene und das JZ-Volumen (JZV) zu ermitteln. Die Ratio JZV/EV wurde berechnet. Diese Parameter wurden mit dem Ausgang der klinischen Schwangerschaft verglichen.

Ergebnisse Die 3 D-Aufnahme zeigte, dass die JZ eine gute Intra- und Interobserver-Konsistenz erreichte $(k=0,862$; $k=0,694)$. Die Schwangerschaftsrate betrug insgesamt $47 \%$.
Es gab einen hochsignifikanten Unterschied zwischen schwangeren und nicht schwangeren Frauen in Bezug auf Alter $(p<0,001), J Z V(p=0,003)$ und JZV/EV $(p<0,001)$ am Tag vor dem Transfer. Alter und JZV/EV waren unabhängige Faktoren für die Vorhersage des Erfolgs bei IVF-Transfer $(p=0,010$; $p=0,016)$. Die Fläche unter der ROC-Kurve von JZV/EV zur Vorhersage einer klinischen Schwangerschaft betrug 0,688 , der Cut-off-Wert lag bei 0,54. Die Sensitivität betrug 83,8\% und die Spezifität 50,0\%.

Schlussfolgerung Alter und JZV/EV sind unabhängige Faktoren für die Vorhersage eines erfolgreichen Transfers kryokonservierter Embryonen bei IVF. Eine geringere JZV/EV-Ratio war für eine klinische Schwangerschaft günstiger.

\section{Introduction}

The uterine junctional zone (JZ) is composed of smooth muscle cells in the myometrium, which is the layer closest to the endometrium. However, the embryological origin of the JZ is different from that of the outer muscularis. Both the JZ and the endometrium are derived from the mesonephric duct [1]. The JZ can also be affected by estrogen and progesterone and have periodic changes [2]. Thickening of the JZ is related to uterine diseases [3], and its thickness is also related to the endocrine state [4]. The JZ is also a source of uterine contractions in non-pregnant women, causing peristalsis of the endometrium, which is associated with low pregnancy rates in natural and assisted reproductive technology cycles when uterine contractions are excessive and uncoordinated [5]. Hence, it plays an important role in reproduction and pregnancy [6].

The JZ was first described in 1983 as a low-signal band-like structure between the endometrium and myometrium on T2weighted magnetic resonance imaging (MRI) [7]. In 1990, Mitchell et al. compared the display of the JZ on MRI and transvaginal ultrasonography (TVS). Although there was a good correlation between the two methods of measuring the JZ and histological measurements, there was a small difference in the thickness of the JZ measured by TVS and MRI [8], which was related to the different imaging methods. The JZ appears as a compact, smooth muscle bundle, longitudinally parallel to the endometrium. There is an obvious transition from the endometrium to the JZ but the transition from the JZ to the myometrium is not obvious [9]. This also makes it difficult to accurately measure JZ thickness on twodimensional (2D) ultrasound imaging. With the development of three-dimensional (3D) ultrasound, the $3 \mathrm{D}$ imaging mode enhanced the contrast, making the JZ display clearer. In 2012, Naftalin et al. applied a 3D ultrasound coronal plane to display the JZ and achieved good repeatability [8]. However, this only analyzed one section of the JZ and could not reflect the whole picture. Furthermore, the contractile function of the JZ does not exist completely linearly under the endometrium [10]. Therefore, we aimed to analyze the JZ more objectively through the calculation of the $3 \mathrm{D}$ volume and explore its relationship with the failure of frozen embryo transfer cycles in in vitro fertilization (IVF).

\section{Methods}

This was a prospective study. A total of 158 women who underwent IVF were enrolled between January 2020 and January 2021. The criteria for inclusion were frozen embryo transfers, transplantation of more than one high-quality embryo, and a clearly displayed endometrium on ultrasound examination. The exclusion criteria were uterine malformation, adenomyosis, endometriosis, uterine fibroids, hydrosalpinx, and intrauterine adhesions. The patients' medical histories were recorded, including age, type of infertility, and cause of infertility, followed by ultrasound evaluation to identify any exclusion criteria. Ultrasonography was performed on the day before transplantation to measure the endometrial thickness (ET) and to preserve 2-3 3D volume images for offline analysis. The optimal $3 \mathrm{D}$ volume images were used to obtain the volume of the endometrium (EV), the average thickness of the $\mathrm{JZ}$ in the coronal plane, and the volume of the JZ (JZV). The JZV divided by the EV was JZV/EV. The age, number of high-quality embryos, ET, average thickness of the JZ, the JZV, and JZV/EV were compared with the outcomes of clinical pregnancy.

This research program was approved by the Ethics Review Committee. Women who agreed to participate in the study provided informed consent.

\section{$3 \mathrm{D}$ image}

All examinations were performed using a Voluson E10 (GE Healthcare, Zipf, Austria) ultrasound machine (Probe model RIC5-9-D). Patients had to empty their bladder before the examination, and the midsagittal plane of the uterus was used as the starting section for the data collection regarding uterine volume. To reduce artifacts, the entire uterus was included in the $3 \mathrm{D}$ scan, with the probe still, and patients were instructed to hold their breath as much as possible. After data collection, 2-3 uterine volumes were saved per woman for later analysis. Two observers, A and B, performed an offline analysis of the $3 \mathrm{D}$ volume images. $\mathrm{A}$ and $\mathrm{B}$ were doctors with more than 15 years of experience in obstetrics and gynecology ultrasound and more than 5 years in gynecology $3 \mathrm{D}$ ultrasound. 


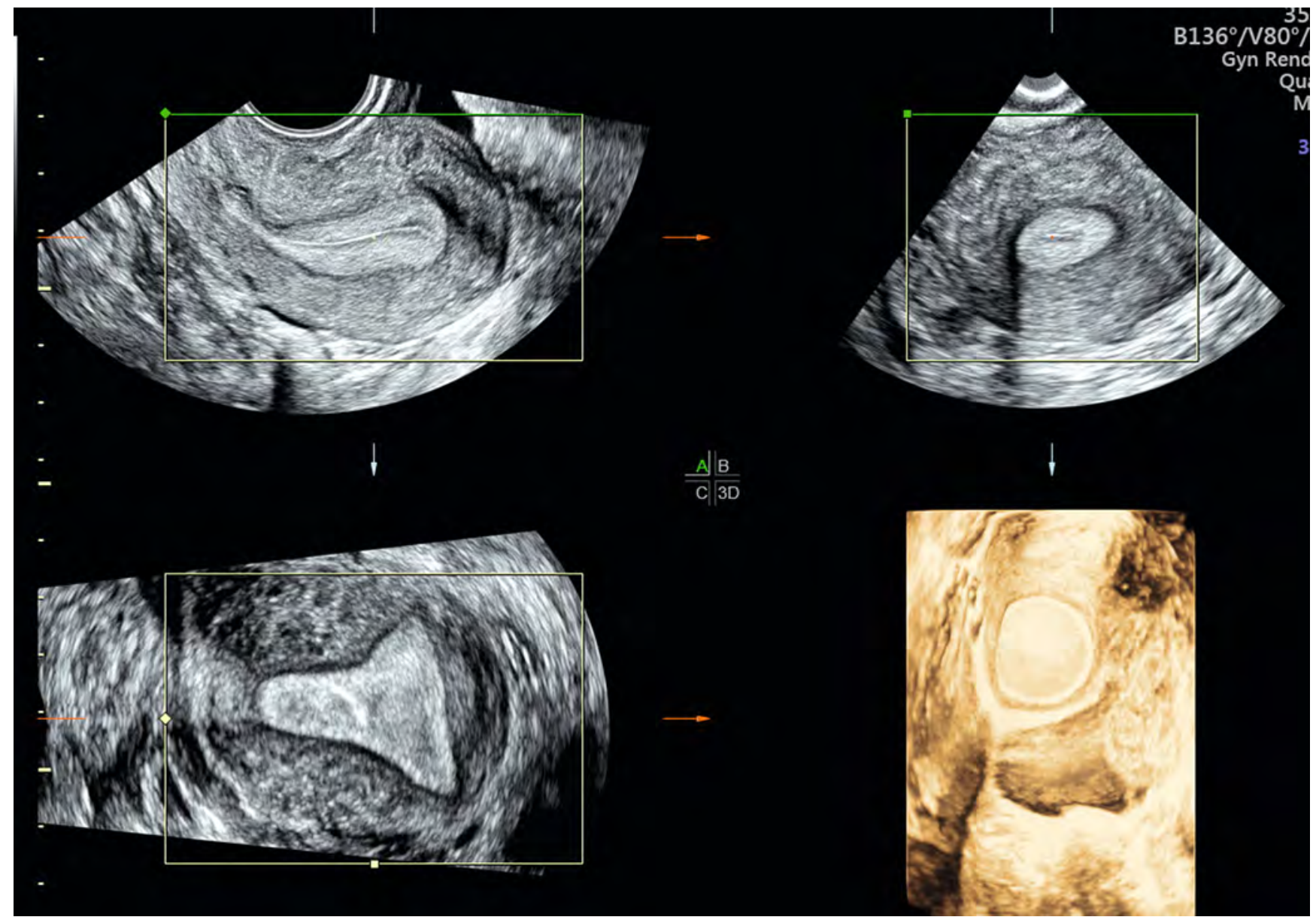

- Fig. 1 In the 3D TVS multi-plane and render view: 3 D uterine volume data is shown in four images. Plane A was a midsagittal section, plane B was a transverse section, and plane $\mathrm{C}$ was a coronal section.

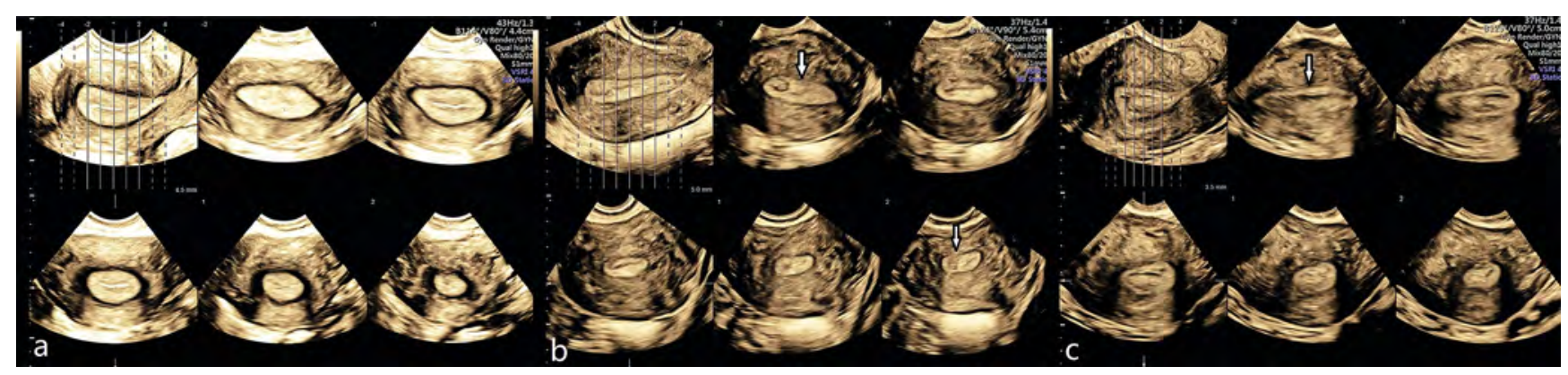

- Fig. 2 In the 3 D TVS multi-plane and TUI view: The JZ is displayed. a JZ is clearly visible on all five transverse sections; score = 1 . $\mathbf{b}$ In sections 1 and 5 , the JZ is not clearly displayed locally; score $=2$ (white arrow). $\mathbf{c}$ In section 1 , the JZ is completely obscured; score $=3$ (white arrow). JZ: junctional zone.

\section{Consistency study of $3 \mathrm{D}$ image observation}

Standardization of methods for $3 \mathrm{D}$ image post-processing and evaluation of JZ display satisfaction were initially performed in a group of 30 patients not included in the study sample. The evaluation method for the degree of satisfaction with the 3D JZ display was as follows: The $3 \mathrm{D}$ volume was shown in four images and finetuned such that plane A was a midsagittal section, plane B was a transverse section, and plane $\mathrm{C}$ was a coronal section ( $\mathbf{F i g . 1}$ ).
For the B plane using multiple TUI VCAD display methods, we chose the $2 \times 3$-plane six-image display mode, adjusted the layer thickness to enable the endometrium to display in the remaining 5 planes, added volume contrast imaging $(\mathrm{VCl})$, selected a layer thickness of $1 \mathrm{~mm}$ and high-contrast mode, and set the image magnification as far as possible under the observation of the JZ, which is the low echogenic band around the endometrium. The display of the JZ was then ranked 1, 2, or 3 ( $\triangleright$ Fig. 2). A score of 1 


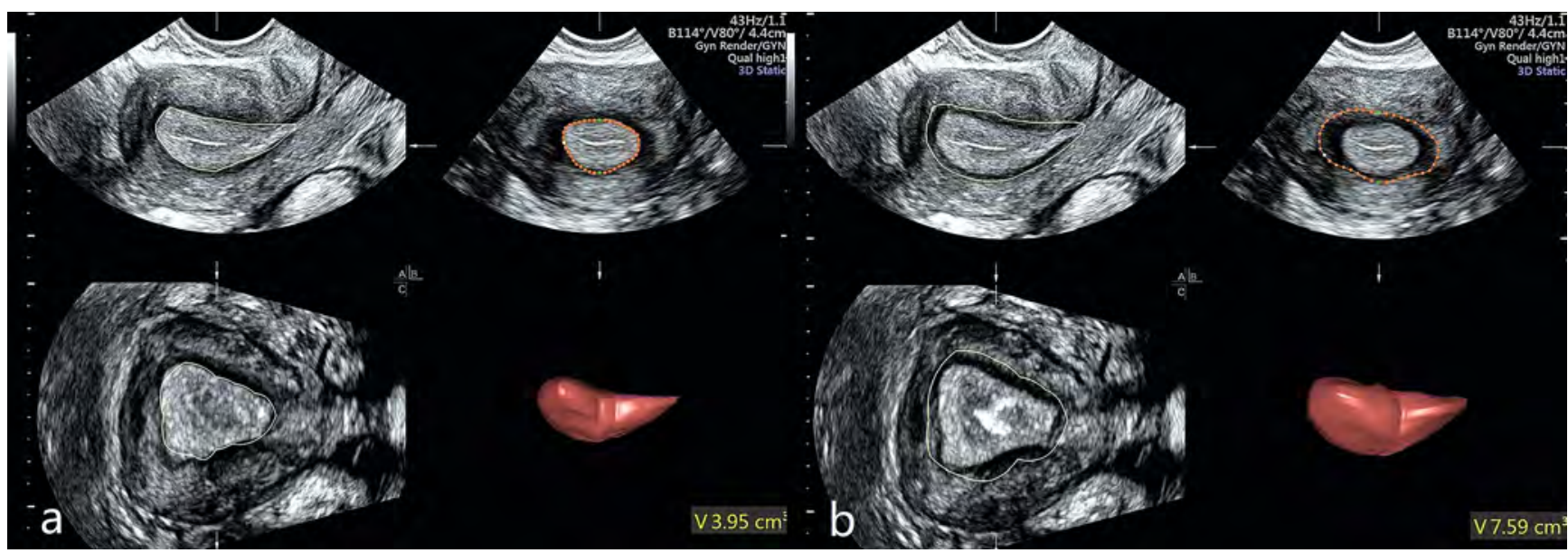

- Fig. 3 In the 3D TVS VOCAL view: Calculation of the JZ volume. a Calculation of the volume of the endometrium (EV); $E V=3.95 \mathrm{~cm}^{3}$. $\mathbf{b}$ Calculation of the volume of the $J Z$ and the endometrium (JZV $+E V) ; J Z V+E V=7.59 \mathrm{~cm}^{3}$. The JZV is obtained by subtracting the EV from the volume of the $\mathrm{JZ}$ and the endometrium; JZV=7.59-3.95 $=3.64 \mathrm{~cm}^{3}$. JZ: junctional zone.

was the best, 2 was satisfactory, and 3 was dissatisfactory. The $3 \mathrm{D}$ volume images were analyzed offline by the two observers and scored. These scores were used to determine interobserver consistency [Kappa (K)]. One month later, observer A performed a second analysis rating on the $3 \mathrm{D}$ images, which was tested for intraobserver consistency with the first score.

A score of 1 (best) indicated that the JZ was clearly visible on all 5 transverse sections ( $\triangleright$ Fig. 2a), 2 (satisfactory) indicated that only 1-2 out of 5 sections of the local JZ could not be clearly displayed ( $\triangleright$ Fig. 2b), and 3 (unsatisfactory) indicated that the JZ was completely unclear in 1 or more of the 5 sections or that the local JZ was not clearly displayed in more than 3 sections ( $\triangleright$ Fig. $2 c$ ).

\section{JZ volume of $3 \mathrm{D}$ image}

The two doctors ( $A$ and $B$ ) independently observed and analyzed the $3 \mathrm{D}$ volumes. Patients who were scored 3 by one of the two observers were excluded. The two doctors calculated the JZV for the patients with scores of 1 and 2 . We then selected volume analysis to start the volume calculation program. Starting from plane $\mathrm{B}$, we marked every $30^{\circ}$ and obtained the EV after completion ( $\vee$ Fig. 3a). The same method was used to carry out another volume calculation along the outer edge of the JZ to obtain JZV+EV ( $\triangleright$ Fig. 3b). The JZV was obtained by subtracting the EV from JZV $+E V$. Two procedures were performed for each patient, and an average was taken.

\section{Average thickness of the $\mathrm{JZ}$ in the coronal plane}

The $3 \mathrm{D}$ multi-plane method was used to image the $3 \mathrm{D}$ volume on the $C$ plane. The $\mathrm{VCl}$ was added, a layer thickness of $1 \mathrm{~mm}$ was selected, and the view was adjusted until the endometrial cavity and extrauterine contour were both satisfactory with the coronal plane image, and the two stromal parts of the fallopian tube were shown. In the high-contrast mode, the JZ can be seen as a hypoechoic area around the endometrium. The maximum and minimum values of the JZ on the two lateral walls and the bottom wall were measured for the coronal plane with the most satisfac- tory display, and the values were added and divided by 6 to obtain the average value of the coronal plane ( $\triangleright$ Fig. 4 ).

\section{Clinical pregnancy criteria}

Ultrasound was performed on the patients at 6 weeks of gestation, and in cases of the existence of a fetal heart rate, the patient was diagnosed with clinical pregnancy [11].

\section{Statistical analysis}

SPSS software (version 26.0; IBM Corporation, Armonk, NY, USA) was used for statistical analysis. Measurement data are expressed as mean \pm standard deviation. The kappa ( $\mathrm{k}$ ) test was used for the consistency study. Univariate logistic regression analysis was performed for all parameters and clinical pregnancy, and multivariate logistic regression analysis was performed on the significant parameters of the results. Receiver operating characteristic (ROC) curves were used to determine the predictive value of the valuable parameters. Statistical significance was set at $p<0.05$.

\section{Results}

A consistency study of the JZ on $3 \mathrm{D}$ ultrasound was conducted on 30 women who met the inclusion criteria. The two observers mutually agreed with their judgments on the JZ display. The interobserver consistency was $\mathrm{K}=0.694$ and the $95 \%$ confidence interval $(\mathrm{Cl})$ was $0.431-0.957$. The intraobserver consistency was $\mathrm{K}=0.862$ and the $95 \% \mathrm{Cl}$ was 0.669-1.056 ( $\vee$ Table 1, $\triangleright$ Table 2,

\section{- Table 3).}

A total of 158 eligible cases were included in this study, 8 of which were subsequently excluded ( 6 cases of ET< $6 \mathrm{~mm}$ or ET> $16 \mathrm{~mm}, 2$ cases of failed embryo resuscitation. The JZ showed unsatisfactory results, with a score of 3 , and another five cases were excluded. Subsequently, three cases of early spontaneous abortion caused by chromosomal abnormalities were excluded. Finally, the data of 142 patients were analyzed ( $\triangleright$ Fig. 5). The characteristics of the study population are shown in $>$ Table 4. 


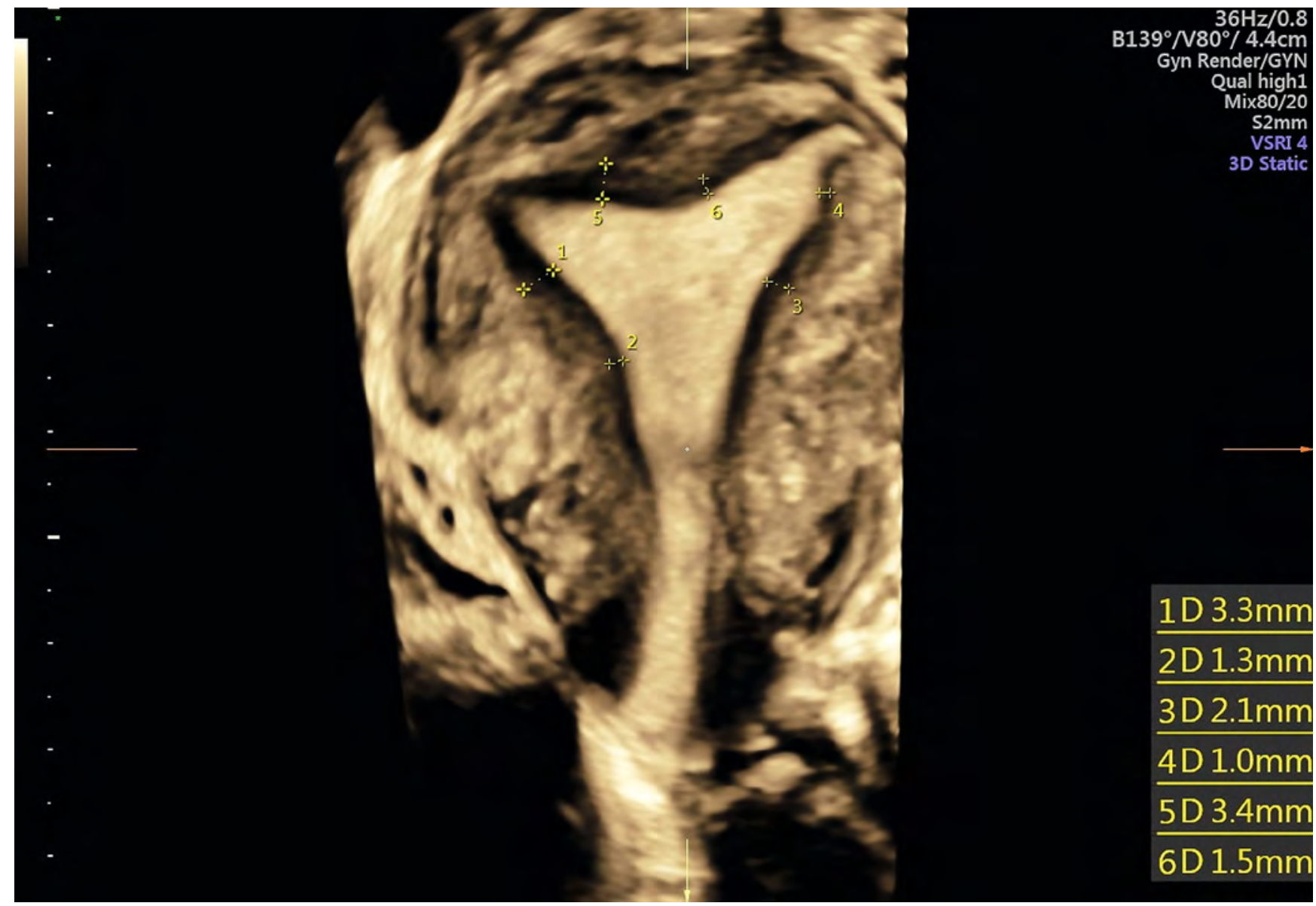

- Fig. 4 In the 3D TVS multi-plane view: Calculation of the average JZ thickness in the coronal plane $(\mathrm{mm})$. The maximum and minimum JZ values on the two lateral walls and the bottom wall were measured, and the values were added and divided by 6 to get the average value of the coronal plane $[(3.3+1.3+2.1+1.0+3.4+1.5) / 6=2.1]$. The average $J Z$ thickness was $2.1 \mathrm{~mm}$. JZ: junctional zone.

- Table 1 Interobserver variability in assessment of JZ.

\begin{tabular}{|l|l|l|l|l|l|}
\hline & \multicolumn{3}{|c|}{ Observer B } & & Total \\
\hline & & $\mathbf{1}$ & $\mathbf{2}$ & $\mathbf{3}$ & \\
\hline \multirow{2}{*}{ Observer A } & 1 & 19 & 0 & 0 & 19 \\
\hline & 2 & 5 & 4 & 0 & 9 \\
\hline & 3 & 0 & 0 & 2 & 2 \\
\hline Total & & 24 & 4 & 2 & 30 \\
\hline
\end{tabular}

JZ: junctional zone.

Among the 142 patients, 68 were pregnant, and the clinical pregnancy rate was $47 \%$ ( $>$ Table 5 ). Univariate analysis was performed on age, number of high-quality embryos, ET, EV, average thickness of the JZ, JZV, and JZV/EV of the 142 patients ( $\triangleright$ Table 6). Age, JZV, and JZV/EV were included in the multivariate analysis. Multivariate analysis showed that age and JZV/EV were in-
- Table 2 Intraobserver variability in assessment of JZ for observer A.

\begin{tabular}{|l|l|l|l|l|l|}
\hline & \multicolumn{3}{|c|}{ Observer A } & Total \\
\hline & & $\mathbf{1}$ & $\mathbf{2}$ & $\mathbf{3}$ & \\
\hline \multirow{2}{*}{ Observer A } & 1 & 22 & 0 & 0 & 22 \\
\hline & 2 & 2 & 4 & 0 & 6 \\
\hline Total & 3 & 0 & 0 & 2 & 2 \\
\hline & & 24 & 4 & 2 & 30 \\
\hline JZ: junctional zone. & & & & \\
\hline
\end{tabular}

dependent predictors of clinical pregnancy ( $>$ Fig. 6). ROC curve analysis of JZV/EV prediction of clinical pregnancy was performed ( $\triangleright$ Fig. 7). The area under the curve was 0.688 , the cut-off value of JZV/EV for predicting clinical pregnancy was 0.54 , the sensitivity was $83.8 \%$, and the specificity was $50.0 \%$. 
- Table 3 Kappa analysis of inter- and intraobserver variability in assessment of JZ ( $n=30$ for each).

\begin{tabular}{|c|c|c|c|c|c|c|}
\hline & Kappa Value & $\begin{array}{l}\text { Asymptotic } \\
\text { Standard } \\
\text { Error }^{\mathrm{a}}\end{array}$ & $\mathbf{Z}$ & P-value & $\begin{array}{l}\text { Lower } 95 \% \\
\text { Asymptotic } \\
\text { Cl Bound }\end{array}$ & $\begin{array}{l}\text { Upper } 95 \% \\
\text { Asymptotic } \\
\text { Cl Bound }\end{array}$ \\
\hline Interobserver variability & 0.694 & 0.134 & 4.849 & 0.000 & 0.431 & 0.957 \\
\hline Intraobserver variability & 0.862 & 0.099 & 5.694 & 0.000 & 0.669 & 1.056 \\
\hline
\end{tabular}

- Table 4 Participant characteristics and causes of infertility.

\begin{tabular}{|l|l|}
\hline Parameter & $\mathbf{N}=\mathbf{1 4 2}$ \\
\hline Age (years) & $35.28 \pm 4.53$ \\
\hline Duration of infertility (years) & $4.35 \pm 2.47$ \\
\hline Body mass index (kg/m²) & $22.93 \pm 3.58$ \\
\hline Basal serum FSH (IU/L) & $7.98 \pm 4.50$ \\
\hline Basal serum LH (IU/L) & $5.86 \pm 3.06$ \\
\hline Basal serum E2 level (p g/mL) & $42.34 \pm 16.69$ \\
\hline - Male infertility & $20 / 142(14.08 \%)$ \\
\hline - ovulatory dysfunction & $38 / 142(26.76 \%)$ \\
\hline - tubal infertility & $48 / 142(33.80 \%)$ \\
\hline - unexplained infertility & $36 / 142(25.35 \%)$ \\
\hline
\end{tabular}

FSH: follicle-stimulating hormone; LH: luteinizing hormone; E2: estradiol.

\section{Discussion}

The JZ has been studied for nearly 40 years, and there are still many ambiguities [12]. The JZ can be detected and evaluated using T2-weighted MRI and TVS [13]. The development of $3 \mathrm{D}$ ultrasound imaging technology has made the study of the JZ more convenient and extensive.

In this study, inter- and intraobserver consistencies were first studied. We then established a $3 \mathrm{D}$ multi-plane and TUI method and used five transverse sections to evaluate the display of the JZ. This overcame the shortcomings of the single coronal plane display in previous studies [6]. In the subsequent volume calculation, we chose the B-plane for tracing and calculation, which was concordant with the consistency study, to reduce the errors in the analysis. In the analysis of 30 cases of $3 \mathrm{D}$ volume, there was good inter- and intraobserver consistency in the judgment of the JZ display ( $K=0.694$ and $K=0.862$, respectively), and the results were similar to those of Naftalin et al.'s study [14]. Of the 30 patients in this study, 2 were assigned a score of 3, which was agreed upon by the two observers. The differences between the observers were mainly reflected in the judgments of scores 1 and 2, which did not affect the cases with scores 1 and 2 in the analysis

- Table 5 Comparison of clinical characteristics and ultrasonic parameters between the clinical pregnancy group and non-clinical pregnancy group.

\begin{tabular}{|c|c|c|c|c|}
\hline Variable & $\begin{array}{l}\text { Pregnancy } \\
(n=68)\end{array}$ & $\begin{array}{l}\text { Non-pregnancy } \\
(n=74)\end{array}$ & $\mathbf{t}$ & $\mathbf{P}$ \\
\hline Age (years) & $33.81 \pm 3.75$ & $36.64 \pm 4.78$ & 3.933 & $<0.001$ \\
\hline Number of high-quality embryos & $1.47 \pm 0.50$ & $1.51 \pm 0.56$ & 0.482 & 0.631 \\
\hline $\mathrm{ET}(\mathrm{mm})$ & $10.13 \pm 2.21$ & $9.74 \pm 2.49$ & 0.981 & 0.328 \\
\hline $\mathrm{EV}\left(\mathrm{cm}^{3}\right)$ & $3.65 \pm 1.37$ & $3.29 \pm 1.48$ & 1.507 & 0.134 \\
\hline $\mathrm{EV}+\mathrm{JZV}\left(\mathrm{cm}^{3}\right)$ & $6.80 \pm 2.13$ & $7.18 \pm 2.63$ & 0.945 & 0.346 \\
\hline $\mathrm{JZV}\left(\mathrm{cm}^{3}\right)$ & $3.15 \pm 1.11$ & $3.89 \pm 1.74$ & 3.052 & 0.003 \\
\hline JZV/EV & $0.47 \pm 0.09$ & $0.55 \pm 0.12$ & 4.315 & $<0.001$ \\
\hline Average thickness of JZ (mm) & $2.59 \pm 0.80$ & $2.66 \pm 0.85$ & 0.546 & 0.586 \\
\hline
\end{tabular}


Female eligible for inclusion $n=158$

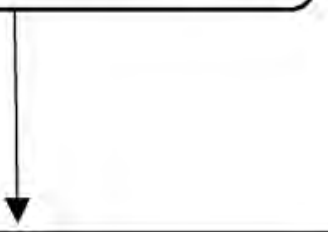

Ultrasonography was performed the

day before transplantation

Canceled the transplant $n=8$

- $\mathrm{ET}<6 \mathrm{~mm}$ or $\mathrm{ET}>16 \mathrm{~mm} \mathrm{n}=6$

- Failure to resuscitate embryos $n=2$

Enter the next day of embryo transfer procedure $n=150$

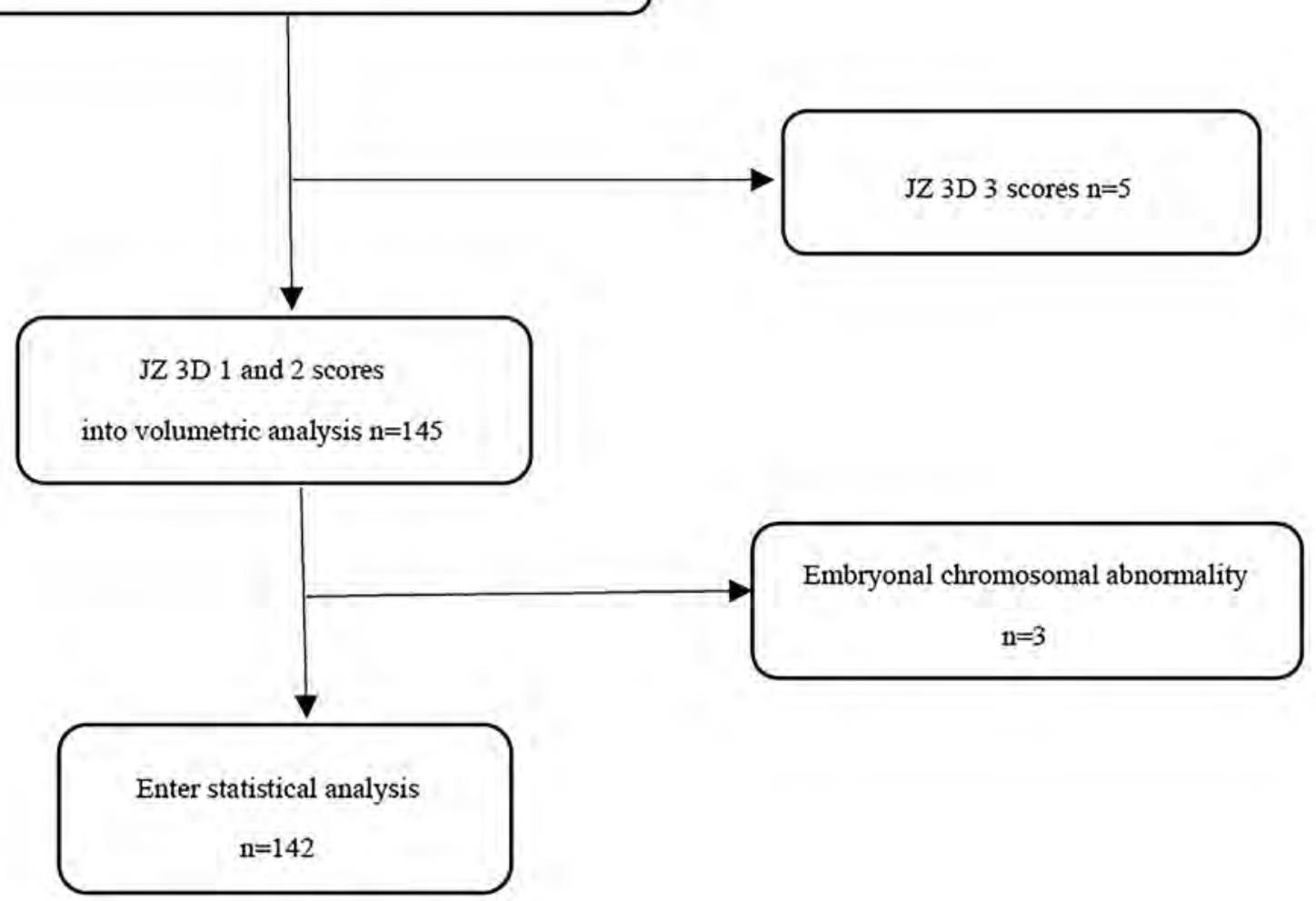

- Fig. 5 Flowchart of the study group.

group. In this study, patients were able to obtain a higher consistency, but this was because they were screened according to the inclusion criteria required for the study, and patients with unsatis- factory uterine cavity images and uterine diseases, such as uterus in horizontal position, uterine malformations, uterine fibroids, were excluded. This enabled the JZ to have a better display and 
- Table 6 Logistic univariate analysis of each parameter.

\begin{tabular}{|l|l|l|}
\hline Variable & Univariate analysis & \\
\hline & OR (95\% CI) & $\mathbf{P}$ \\
\hline Age (years) & $0.860(0.793-0.934)$ & $<0.001$ \\
\hline $\begin{array}{l}\text { Number of high-quality } \\
\text { embryos }\end{array}$ & $0.857(0.458-1.602)$ & 0.628 \\
\hline ET (mm) & $1.073(0.932-1.236)$ & 0.326 \\
\hline EV (cm $\left.{ }^{3}\right)$ & $1.196(0.946-1.512)$ & 0.135 \\
\hline EV + JZV (cm $\left.{ }^{3}\right)$ & $0.935(0.813-1.075)$ & 0.345 \\
\hline JZV (cm $\left.{ }^{3}\right)$ & $0.691(0.532-0.897)$ & 0.005 \\
\hline JZV/EV & $0.001(0.000-0.038)$ & $<0.001$ \\
\hline $\begin{array}{l}\text { Average thickness of JZ } \\
(\mathrm{mm})\end{array}$ & $0.893(0.595-1.339)$ & 0.584 \\
\hline
\end{tabular}

OR: odds ratio; Cl: confidence interval; ET: endometrial thickness; EV: volume of endometrium; JZV: volume of junctional zone; JZ: junctional zone.

achieve a relatively high consistency. Regarding the measurement of the average JZ value on $3 \mathrm{D}$ coronal section, we did not conduct a consistency study because several previous studies have confirmed that this method has good consistency [15].

Embryotic quality and endometrial receptivity (ER) are important factors affecting the success of IVF, of which endometrial factors account for $60 \%$ and embryonic factors account for $40 \%$ [16]. The ET, shape, volume, and blood flow were all considered as ultrasonic indicators of ER. However, different studies on these indicators have conflicting conclusions [17]. Therefore, the ER of ultrasound is still being studied. All patients in this study underwent frozen embryo transfer cycles with more than one high-quality embryo. After statistical analysis, the number of high-quality embryos transplanted was not related to clinical pregnancy $(P=0.631)$. Embryonic factors were excluded, and ER was the focus.

In the past, most MRI studies on the JZ focused on adenomyosis [18]. However, with the deepening of research on the function of the JZ, it has been recognized that it is associated with infertility [19]. Maubon et al. used MRI to study the predictive effect of the JZ on IVF success [20] and showed that JZ thickening was a negative predictor of IVF. However, MRI is not suitable for every patient undergoing IVF because of its high cost and long examination time. Ultrasonography has the advantages of convenience and low cost. As $3 \mathrm{D}$ ultrasound imaging technology is becoming increasingly accurate, many studies have attempted to use it to evaluate the JZ [21, 22]. Maged et al. used 3D ultrasound to observe the $J Z$ and concluded that embryo transplants are more likely to be successful in women with a thin JZ. The higher the maximum value of the JZ, the more likely it is that implantation will fail [23]. However, the study measured the JZ on a coronal section, which may have overlooked the changes in the JZ in other sections ( $\triangleright$ Fig. 8). The 3D multi-plane VOCAL imaging technology can

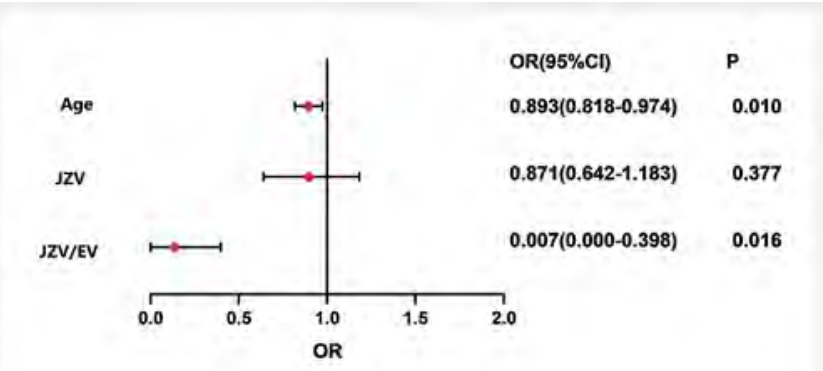

- Fig. 6 Logistic multivariate analysis of age, JZV, JZV/EV, and clinical pregnancy. JZV: volume of the junctional zone; EV: volume of the endometrium.

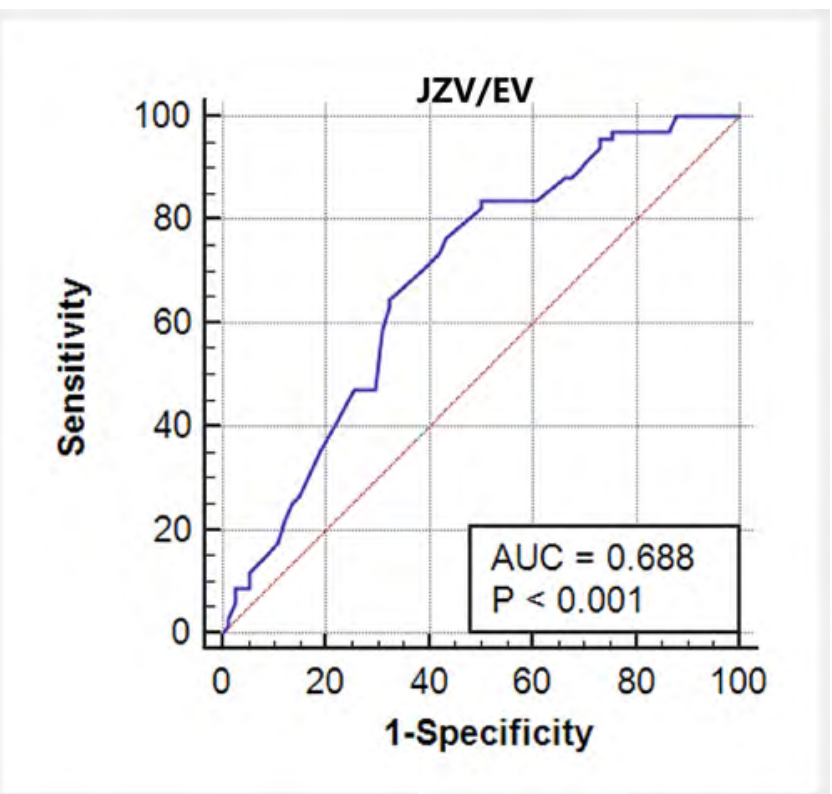

- Fig. 7 Receiver operating characteristic curve of JZV/EV and clinical pregnancy. JZV: volume of the junctional zone; EV: volume of the endometrium.

display multiple planes and ensure more accurate volume measurements. Therefore, we want to calculate the JZ volume by tracing multiple planes to evaluate the $\mathrm{JZ}$ more comprehensively. Furthermore, considering the same origin of the $\mathrm{JZ}$ and endometrium, the EV was combined with the JZV for analysis. The results showed that age, JZV, and JZV/EV were all factors that affected the outcome of IVF. However, in the subsequent multivariate logistic analysis, only age and JZV/EV were shown to be independent predictors of clinical pregnancy. The cut-off value of JZV/EV for predicting clinical pregnancy was 0.54 , with a sensitivity of $83.8 \%$ and specificity of $50.0 \%$. None of the 10 women with JZV/ EV $>0.70$ were clinically pregnant.

In contrast to previous findings, in our study, the average JZ value was not a predictor of IVF success. This may be because cases of adenomyosis and endometriosis were excluded from $2 \mathrm{D}$ ultrasound. Patients with changes in the JZ alone were not diagnosed as adenomyosis because the diagnosis had not yet been fi- 


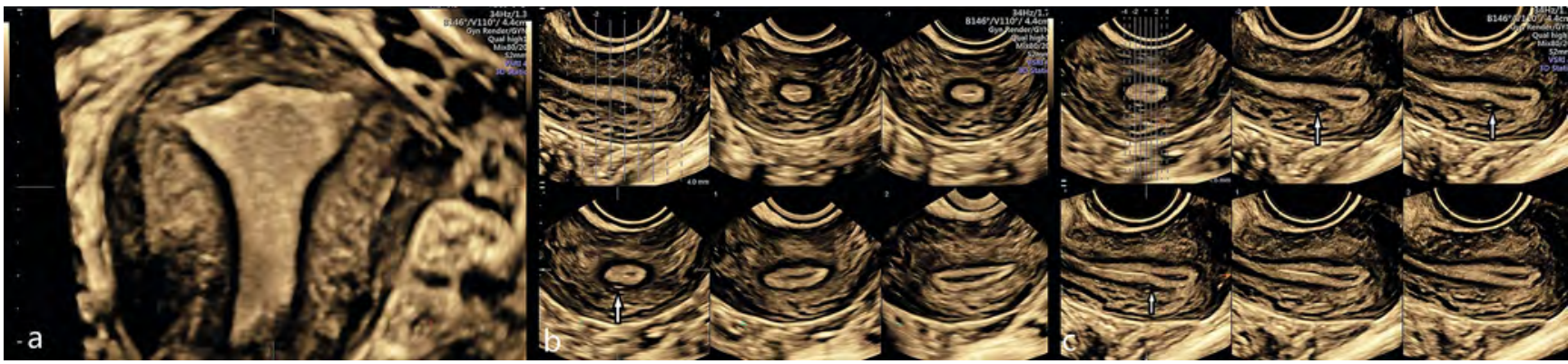

- Fig. 8 In the 3D TVS multi-plane and TUI view: Local thickening of the JZ sometimes was not shown only in the coronal plane. a) The JZ showed uniform thickness on the coronal plane. b) Local thickening of the JZ was shown in the transverse sections in the 3D TUI view (white arrow). c) Local thickening of the JZ was shown in the longitudinal sections in the $3 \mathrm{D}$ TUI view (white arrow).

nalized [24], and they were only considered as changes in the JZ. Therefore, the difference in the JZ of patients in this group was not significant, which may be the reason why the average JZ value in the coronal plane in $3 \mathrm{D}$ ultrasound in this group did not become an indicator for predicting clinical pregnancy. The JZ volume measurement increased this difference. Hence, the JZV became a predictor, while JZV/EV also added the endometrial factor and thus became a better predictor of IVF success.

Our study used the $\mathrm{JZ}$ volume instead of the maximum and average JZ value on a single ultrasound section in previous studies. To the best of our knowledge, it is the first to combine the JZ volume with the EV for predicting the outcome of IVF. It also reduced the effect of uterine size on the EV and the JZV. However, we still have some limitations. In this study, patients with ET > $16 \mathrm{~mm}$ and $<6 \mathrm{~mm}$ were excluded because these patients would be advised to avoid undergoing transplantation. In addition, the principle of ultrasound imaging is different from that of MRI. Even though $3 \mathrm{D}$ ultrasound may have different JZ displays due to its different imaging modes and image adjustments, the standardization of $3 \mathrm{D}$ ultrasound imaging remains to be further studied. Finally, the relationship between the JZ in ultrasound images and its histology has not been demonstrated in this study, which may become the topic of our future study.

\section{Conclusion}

$\mathrm{JZV} / \mathrm{EV}$ is an independent factor for predicting the success of IVF frozen embryo transfer cycles. A smaller JZV/EV was more beneficial for clinical pregnancy.

Funding

This study was supported by grants from the Guide Project for Key Research and Development Foundation of Liaoning Province (No. 2019]H8/ 10300008), Scientific Research Project of the Educational Department of Liaoning Province (ZF2019023),345 Talent Project and Liaoning BaiQianWan Talents Program.

\section{Conflict of Interest}

The authors declare that they have no conflict of interest.

\section{References}

[1] Noe M, Kunz G, Herbertz M et al. The cyclic pattern of the immunocytochemical expression of oestrogen and progesterone receptors in human myometrial and endometrial layers: characterization of the endometrial-subendometrial unit. Hum Reprod 1999; 14: 190-197. doi:10.1093/humrep/14.1.190

[2] Wiczyk HP, Janus CL, Richards C] et al. Comparison of magnetic resonance imaging and ultrasound in evaluating follicular and endometrial development throughout the normal cycle. Fertil Steril 1988; 49: 969972. doi:10.1016/s0015-0282(16)59946-4

[3] Mark AS, Hricak H, Heinrichs LW et al. Adenomyosis and leiomyoma: differential diagnosis with MR imaging. Radiology 1987; 163: 527-529. doi:10.1148/radiology.163.2.3562836

[4] McCarthy S, Tauber C, Gore J. Female pelvic anatomy: MR assessment of variations during the menstrual cycle and with use of oral contraceptives. Radiology 1986; 160: 119-123. doi:10.1148/radiology.160.1.3715022

[5] Lesny P, Killick SR. The junctional zone of the uterus and its contractions. An International Journal of Obstetrics \& Gynaecology 2004; 111: 11821189. doi:10.1111/j.1471-0528.2004.00350.x

[6] Silva V, Ramos FF, Brás AFM et al. Junctional Zone in Infertile Women: A Three-dimensional Ultrasound Study. Rev Bras Ginecol Obstet 2020; 42: 152-159. doi:10.1055/s-0040-1708089

[7] Hricak H, Alpers C, Crooks LE et al. Magnetic resonance imaging of the female pelvis: initial experience. Am J Roentgenol 1983; 141: 11191128. doi:10.2214/ajr.141.6.1119

[8] Mitchell DG, Schonholz L, Hilpert PL et al. Zones of the uterus: discrepancy between US and MR images. Radiology 1990; 174: 827-831

[9] Brown HK, Stoll BS, Nicosia SV et al. Uterine junctional zone: correlation between histologic findings and MR imaging. Radiology 1991; 179: 409-413

[10] Tanos V, Lingwood L, Balami S. The importance of the junctional zone of the endometrium in human reproduction. Hum Fertil (Camb) 2020; 6: 1-9. doi:10.1080/14647273.2020.1720316

[11] Mercé LT, Barco MJ, Bau S et al. Are endometrial parameters by threedimensional ultrasound and power Doppler angiography related to in vitro fertilization/embryo transfer outcome? Fertil Steril 2008; 89: 111117. doi:10.1016/j.fertnstert.2007.02.029

[12] Tanos V, Lingwood L, Balami S. Junctional Zone Endometrium Morphological Characteristics and Functionality: Review of the Literature. Gynecol Obstet Invest 2020; 85: 107-117. doi:10.1159/000505650

[13] Sudderuddin S, Helbren E, Telesca M et al. MRI appearances of benign uterine disease. Clin Radiol 2014; 69: 1095-1104. doi:10.1016/j. crad.2014.05.108 
[14] Naftalin J, Hoo W, Nunes $\mathrm{N}$ et al. Inter- and intraobserver variability in three-dimensional ultrasound assessment of the endometrial-myometrial junction and factors affecting its visualization. Ultrasound Obstet Gynecol 2012; 39: 587-591. doi:10.1002/uog.10133

[15] Rasmussen CK, Glavind J, Madsen LD et al. Repeatability of Junctional Zone Measurements Using 3-Dimensional Transvaginal Sonography in Healthy Fertile Women. J Ultrasound Med 2016; 35: 1497-1508. doi:10.7863/ultra.15.06086

[16] Al Chami A, Saridogan E. Endometrial Polyps and Subfertility. J Obstet Gynaecol India 2017; 67: 9-14. doi:10.1007/s13224-016-0929-4

[17] Craciunas L, Gallos I, Chu J et al. Conventional and modern markers of endometrial receptivity: a systematic review and meta-analysis. Hum Reprod Update 2019; 25: 202-223. doi:10.1093/humupd/dmy044

[18] Novellas S, Chassang M, Delotte J et al. MRI characteristics of the uterine junctional zone: from normal to the diagnosis of adenomyosis. Am J Roentgenol 2011; 196: 1206-1213

[19] Brosens I, Derwig I, Brosens ] et al. The enigmatic uterine junctional zone: the missing link between reproductive disorders and major ob- stetrical disorders? Hum Reprod 2010; 25: 569-574. doi:10.1093/humrep/dep474

[20] Maubon A, Faury A, Kapella M et al. Uterine junctional zone at magnetic resonance imaging: a predictor of in vitro fertilization implantation failure. J Obstet Gynaecol Res 2010; 36: 611-618. doi:10.1111/j.14470756.2010.01189.x

[21] Exacoustos C, Luciano D, Corbett B et al. The uterine junctional zone: a 3-dimensional ultrasound study of patients with endometriosis. Am J Obstet Gynecol 2013; 209: 1-7

[22] Barbanti C, Centini G, Lazzeri L et al. Adenomyosis and infertility: the role of the junctional zone. Gynecol Endocrinol 2021; 15: 1-7. doi:10.1080/ 09513590.2021 .1878131

[23] Maged AM, Ramzy AM, Ghar MA et al. 3D ultrasound assessment of endometrial junctional zone anatomy as a predictor of the outcome of ICSI cycles. Eur J Obstet Gynecol Reprod Biol 2017; 212: 160-165. doi:10.1016/j.ejogrb.2017.03.035

[24] Bazot M, Daraï E. Role of transvaginal sonography and magnetic resonance imaging in the diagnosis of uterine adenomyosis. Fertil Steril 2018; 109: 389-397. doi:10.1016/j.fertnstert.2018.01.024 